\title{
THE Ly $\alpha$-LyC CONNECTION: EVIDENCE FOR AN ENHANCED CONTRIBUTION OF UV-FAINT GALAXIES TO COSMIC REIONIZATION
}

\author{
Mark Dijkstra $^{1}$, Max Gronke $^{1}$, and Aparna Venkatesan ${ }^{2}$ \\ ${ }^{1}$ Institute of Theoretical Astrophysics, University of Oslo, P.O. Box 1029 Blindern, NO-0315 Oslo, Norway; mark.dijkstra@astro.uio.no \\ 2 Department of Physics and Astronomy, University of San Francisco, 2130 Fulton Street, San Francisco, CA 94117, USA \\ Received 2016 April 13; revised 2016 June 7; accepted 2016 June 29; published 2016 September 2
}

\begin{abstract}
The escape of ionizing Lyman continuum (LyC) photons requires the existence of low- $N_{\mathrm{H}_{\mathrm{I}}}$ sightlines, which also promote escape of Ly $\alpha$. We use a suite of 2500 Ly $\alpha$ Monte-Carlo radiative transfer simulations through models of dusty, clumpy interstellar ("multiphase") media from Gronke \& Dijkstra, and compare the escape fractions of Ly $\alpha$ $\left(f_{\mathrm{esc}}^{\mathrm{Ly} \alpha}\right)$ and LyC radiation $\left(f_{\mathrm{esc}}^{\mathrm{ion}}\right)$. We find that $f_{\mathrm{esc}}^{\text {ion }}$ and $f_{\mathrm{esc}}^{\mathrm{Ly} \alpha}$ are correlated: galaxies with a low $f_{\mathrm{esc}}^{\mathrm{Ly} \alpha}$ consistently have a low $f_{\mathrm{esc}}^{\text {ion }}$, while galaxies with a high $f_{\mathrm{esc}}^{\mathrm{Ly} \alpha}$ exhibit a large dispersion in $f_{\mathrm{esc}}^{\text {ion }}$. We argue that there is increasing observational evidence that $\operatorname{Ly} \alpha$ escapes more easily from UV-faint galaxies. The correlation between $f_{\mathrm{esc}}^{\text {ion }}$ and $f_{\text {esc }}^{\mathrm{Ly} \alpha}$ then implies that UV-faint galaxies contribute more to the ionizing background than implied by the faint-end slope of the UV luminosity function. In multiphase gases, the ionizing escape fraction is most strongly affected by the cloud covering factor, $f_{\mathrm{cl}}$, which implies that $f_{\text {esc }}^{\text {ion }}$ is closely connected to the observed Ly $\alpha$ spectral line shape. Specifically, LyC-emitting galaxies typically having narrower, more symmetric line profiles. This prediction is qualitatively similar to that for "shell models."
\end{abstract}

Key words: dark ages, reionization, first stars - galaxies: high-redshift - intergalactic medium - line: profiles radiative transfer - ultraviolet: galaxies

\section{INTRODUCTION}

The escape fraction of ionizing photons, $f_{\mathrm{esc}}^{\text {ion }}$, represents one of the key parameters describing cosmic reionization (e.g., Cen 2003; Haiman \& Holder 2003; Wyithe \& Loeb 2003; Mitra et al. 2011). Observational constraints on $f_{\mathrm{esc}}^{\text {ion }}$ are still weak (see Figure 13 of Smith et al. 2016). Ionizing photons, also known as Lyman continuum (LyC) photons, have only been directly observed to escape for a handful of galaxies (e.g., Borthakur et al. 2014; Izotov et al. 2016; Vanzella et al. 2016b, also see Benson et al. 2013; Smith et al. 2016 and references therein). Observations of the Ly $\alpha$ forest constrain the $\mathrm{LyC}$ volume emissivity (the rate at which LyC photons are released into the intergalactic medium (IGM) per unit volume), while observations of the UV luminosity function of star-forming galaxies provide direct constraints on the production rate of LyC photons. These two constraints combined constrain the volume-averaged escape fraction of ionizing photons, denoted by $\left\langle f_{\text {esc }}^{\text {ion }}\right\rangle$, and show that $\left\langle f_{\text {esc }}^{\text {ion }}\right\rangle$ increases with redshift (Inoue et al. 2006; Kuhlen \& Faucher-Giguère 2012; Becker \& Bolton 2013).

The LyC escape fraction depends on more than just redshift. Various models and simulations predict that $f_{\text {esc }}^{\text {ion }}$ decreases with the mass of the dark matter halo (e.g., Yajima et al. 2011; Ferrara \& Loeb 2013; Paardekooper et al. 2013; Wise et al. 2014, but also see Gnedin et al. 2008; Ma et al. 2015; Sharma et al. 2016), which in turn correlates with observables such as the non-ionizing UV-continuum luminosity of galaxies. The reason why not all simulations agree on this mass dependence is partly because different studies focus on galaxies with very different masses, at very different redshifts, and different implementations for sub-grid physics associated with feedback, which can strongly affect the properties of the simulated interstellar medium (ISM). Ab initio modeling of $f_{\text {esc }}^{\text {ion }}$ still represents a major theoretical challenge (see, e.g., Fernandez \& Shull 2011 and Paardekooper et al. 2011 for a discussion), and models may have to include additional physical processes such as X-ray heating/ionization (Benson et al. 2013), runaway stars (Conroy \& Kratter 2012), and binary evolution (Ma et al. 2016), all of which can facilitate the escape of ionizing photons.

Irrespective of theoretical and observational uncertainties, the escape of ionizing photons requires that paths exist which contain low column densities of atomic hydrogen, i.e., $N_{\mathrm{H} \mathrm{I}}<1 / \sigma_{\text {ion }} \approx 10^{17} \mathrm{~cm}^{-2}$, where $\sigma_{\text {ion }}=6 \times 10^{-18} \mathrm{~cm}^{2}$ denotes the photoionization cross section evaluated at the Lyman limit (e.g., Verner et al. 1996). These same paths of low column density provide escape routes for Ly $\alpha$ photons (Behrens et al. 2014; Verhamme et al. 2015). The escape of $\mathrm{LyC}$ and $\mathrm{Ly} \alpha$ are therefore expected to be correlated, at least at some level (e.g., Rauch et al. 2011; Erb et al. 2014; Micheva et al. 2016). If the escape of LyC photons is facilitated by (supernova-driven) winds that blew holes of low column density (see, e.g., Dove et al. 2000, Sharma et al. 2016), then this provides a physical mechanism connecting $f_{\mathrm{esc}}^{\mathrm{ion}}$ and $f_{\mathrm{esc}}^{\mathrm{Ly} \alpha}$, because observations of $\operatorname{Ly} \alpha$-emitting galaxies indicate that galactic outflows promote the escape of Ly $\alpha$ photons (Kunth et al. 1998; Atek et al. 2008; Wofford et al. 2013; RiveraThorsen et al. 2015, see Hayes 2015 for a review).

The goal of this paper is to more quantitatively explore the correlation between $\mathrm{Ly} \alpha$ and $\mathrm{LyC}$ photons, for which we use a large suite of simplified models of the multiphase ISM that span the wide range of physical conditions encountered in observed galaxies (first presented in Gronke \& Dijkstra 2016). Yajima et al. (2014) previously found a clear correlation between $f_{\text {esc }}^{\mathrm{Ly} \alpha}$ and $f_{\text {esc }}^{\text {ion }}$ in their cosmological hydrodynamical simulations of a Milky Way-like galaxy. Their calculations should be viewed as a "bottom-up" (or ab initio) approach to quantifying this correlation, while our work should be viewed as a "top-down" (or empirical) approach. As neither approach has converged yet (see Section 2), our work should be viewed 
as complementary to that of Yajima et al. (2014). Addressing the correlation between $f_{\mathrm{esc}}^{\mathrm{Ly} \alpha}$ and $f_{\mathrm{esc}}^{\mathrm{ion}}$ has become (even) more relevant for cosmic reionization because, as we will argue in Section 4.1, there is increasing evidence that $f_{\mathrm{esc}}^{\mathrm{Ly} \alpha}$ increases toward lower UV luminosities.

The outline of this paper is as follows: we present our models in Section 2 and show the predicted correlation between $f_{\mathrm{esc}}^{\mathrm{Ly} \alpha}$ and $f_{\mathrm{esc}}^{\mathrm{ion}}$ in Section 3. We discuss implications of our results in Section 4, before presenting our conclusions in Section 5 .

\section{THE MODEL}

The escape of both ionizing and Ly $\alpha$ photons depends sensitively on the distribution of neutral gas throughout the interstellar medium. For Ly $\alpha$ photons, the kinematics of this neutral gas is possibly even more important (Kunth et al. 1998; Atek et al. 2008; Steidel et al. 2010; Wofford et al. 2013; Rivera-Thorsen et al. 2015). Modeling Ly $\alpha$ transfer on interstellar scales therefore requires a proper model for both the distribution and kinematics of the neutral gas in the ISM, which likely requires magnetohydrodynamical simulations with subparsec resolution (e.g., Fujita et al. 2009; Dijkstra \& Kramer 2012). This requirement underlines why it is important to have a complementary top-down approach to the bottom-up analysis by Yajima et al. (2014), whose simulations had a spatial resolution of $250 h^{-1}$ comoving pc and gas mass resolution of $M=3 \times 10^{5} h^{-1} M_{\odot}$.

To circumvent the demanding requirements to properly model interstellar Ly $\alpha$ transfer from first principles, this process has been represented by highly simplified models, which include (i) the "shell" model, which consists of a Ly $\alpha$ source surrounded by a geometrically thin shell of neutral, dusty hydrogen, which is (typically) outflowing (see, e.g., Ahn et al. 2003; Verhamme et al. 2006; Gronke et al. 2015a). The shell model-which contains seven free parameters-has been remarkably successful at reproducing observed line profiles of Ly $\alpha$ spectra (e.g., Verhamme et al. 2008; Hashimoto et al. 2015; Yang et al. 2016, although some issues have been pointed out by Barnes \& Haehnelt 2010; Kulas et al. 2012; Chonis et al. 2013). Another simplified model is (ii) the "clumpy ISM" model, which consists of a (large) collection of spherical clumps that contain dusty, neutral hydrogen gas, embedded within a hot interclump medium; the clumps represent simplified versions of multiphase interstellar media (e.g., Neufeld 1991; Hansen \& Oh 2006; Laursen et al. 2013; Gronke \& Dijkstra 2014). Clumpy models naturally give rise to a non-zero porosity of the neutral gas, and a "continuum covering factor" of neutral gas that is less than $100 \%$, both of which facilitate Ly $\alpha$ escape (e.g., Shibuya et al. 2014; RiveraThorsen et al. 2015; Trainor et al. 2015). Both sets of simplified models can be interpreted as "sub-grid" models that describe the Ly $\alpha$ transfer on scales that have not been modeled yet from first principles.

In shell models, the shell completely surrounds the Ly $\alpha$ source. The escape fraction $f_{\mathrm{esc}}^{\text {ion }}$ is determined by its $\mathrm{H}$ I column $\left(N_{\mathrm{HI}}\right)$ as $f_{\mathrm{esc}}^{\text {ion }}(\nu)=\exp \left[-\sigma_{\text {ion }}(\nu) N_{\mathrm{H}}\right]$, and $f_{\text {esc }}^{\text {ion }}$ is practically binary $\left(f_{\mathrm{esc}}^{\text {ion }} \approx 0\right.$ for $N_{\mathrm{H} \mathrm{I}}>10^{17} \mathrm{~cm}^{-2}$ or $f_{\mathrm{esc}}^{\text {ion }} \approx 1.0$ for $N_{\mathrm{H}}<10^{1 \mathrm{~cm}^{-2}}$ ). However, the production rate of $\mathrm{Ly} \alpha$ is zero for $f_{\mathrm{esc}}^{\text {ion }} \approx 1.0$, because nebular luminosities depend on $f_{\text {esc }}^{\text {ion }} \propto\left(1-f_{\text {esc }}^{\text {ion }}\right)($ e.g., Schaerer 2003). The shell model therefore technically only gives rise to $\operatorname{Ly} \alpha$ emission while $f_{\text {esc }}^{\text {ion }} \neq 0$ over a finely tuned narrow range of $N_{\mathrm{H} \text { I }}$ centered on $N_{\mathrm{H} \text { I }} \sim 10^{17} \mathrm{~cm}^{-2}$.

Here, we focus on the clumpy ISM models. We have recently constructed a large library of clumpy models (Gronke \& Dijkstra 2016). In these models, the clumps have H I column densities large enough to make them opaque to LyC photons. However, there exist sightlines that do not penetrate any clumps, and which allow LyC photons to escape. In clumpy models, $f_{\text {esc }}^{\text {ion }}$ is related to the fraction of sightlines from the LyC source(s) that do not intersect any clumps (this corresponds to the "picket fence model" of Heckman et al. 2011).

The geometry of the clumpy ISM model and its main parameters are based on the model described in Laursen et al. (2013), and shown schematically in Figure 1. We refer the interested reader to this paper for a more detailed description of how Laursen et al. (2013) constrain their parameters through observed galaxies. Here, we present only a brief description of the model.

In the clumpy ISM model, the multiphase ISM is represented by a large number of neutral, spherical "clumps" that are embedded within a hot gas. The neutral clumps are distributed in a sphere of radius $r_{\text {gal }}=5 \mathrm{kpc}$. The clouds themselves have radius $r_{\mathrm{cl}}$. The cloud covering factor $f_{\mathrm{cl}}$ denotes the total number of clouds from the center of the sphere to its edge, averaged over all sightlines. The content of the cold [hot] clumps [interclump medium] is described by $T_{\mathrm{cl}}, n_{\mathrm{H} \mathrm{I}, \mathrm{cl}}$ $\left[T_{\mathrm{ICM}}, n_{\mathrm{H} \text { I,ICM }}\right]$ for temperature ${ }^{3}$ and the number density of hydrogen, respectively. The optical depth of the dust through the clouds per path length is given by $\sigma_{d} Z_{\mathrm{cl}} / Z_{\mathrm{Sun}} n_{\mathrm{H} \mathrm{I}}$ where $\sigma_{d}=1.58 \times 10^{-21} \mathrm{~cm}^{2}$ (Pei 1992; Laursen et al. 2009) and $Z_{\mathrm{cl}}$ denotes the "metallicity" of the cloud (the ICM has metallicity $Z_{\mathrm{ICM}} \equiv \zeta_{Z} Z_{\mathrm{cl}}$ ). Following previous analyses, we assume that there is no further structure to the cold clumps. That is, we do not further split up the neutral clumps into "warm" and "cold" neutral media, as is the case for realistic multiphase gases (e.g., McKee \& Ostriker 1977).

The clumps are outflowing ${ }^{4}$ with a velocity profile

$$
v(r)=v_{\infty, \mathrm{cl}}\left\{1-\left(\frac{r}{r_{\min }}\right)^{1-\beta_{\mathrm{cl}}}\right\}^{1 / 2}
$$

for $r>r_{\min }=1 \mathrm{kpc}$ and otherwise zero (Steidel et al. 2010; Laursen et al. 2013). In addition to this, the clouds have a random, isotropic velocity distribution that is Gaussian with a standard deviation $\sigma_{\mathrm{cl}}$.

Ly $\alpha$ photons are emitted randomly following an exponential radial profile of the volume emissivity $\epsilon_{\text {Ly } \alpha}(r)=\mathcal{N} \exp \left(-r / H_{\mathrm{em}}\right)$ where $\mathcal{N}$ is a normalization constant and $r$ is the distance to the center of the cloud. The photon is emitted inside a cloud with probability $P_{\mathrm{cl}}$, which would force it to escape from its birthcloud first. The frequency of the photon is drawn from a Gaussian with standard deviation $\sigma_{i}$. In all our models, we assume that the LyC emission traces Ly $\alpha$ emission exactly, including that a fraction $P_{\mathrm{cl}}$ is emitted

\footnotetext{
3 The temperature is defined as $b^{2} \equiv 2 k_{\mathrm{p}} T / m_{\mathrm{p}}$, where $b^{2}=v_{\mathrm{th}}^{2}+v_{\mathrm{turb}}^{2}$. Here $v_{\text {th }}\left[v_{\text {turb }}\right]$ denotes the thermal [turbulent] velocity of the gas.

4 Changing the sign of $v(r)$ only "flips" the emerging Ly $\alpha$ spectrum around $x=0$, and leaves our $f_{\text {esc }}^{\text {ion }}$ and $f_{\text {esc }}^{\text {Ly } \alpha}$ unaffected.
} 


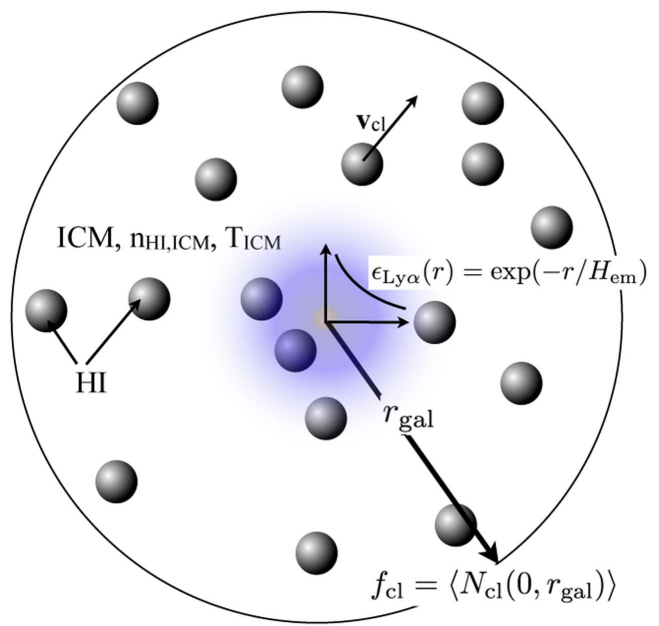

Figure 1. Schematic representation of the adopted geometry in our "clumpy ISM" models, which represent simplified versions of multiphase interstellar media. A sphere or radius $r_{\mathrm{cl}}=5 \mathrm{kpc}$ is filled with outflowing, neutral, dusty clumps of gas embedded within a hot interclump medium. The covering factor $f_{\mathrm{cl}}$ denotes the average number of clumps along sightlines from the center to the edge of the cloud. The clumps surround a spatially extended Ly $\alpha$ (and LyC) source, both of which are characterized by an exponential profile of the volume emissivity with scale length $H_{\mathrm{em}}$. A fraction $P_{\mathrm{cl}}$ of all Ly $\alpha$ and LyC photons are emitted inside cold clumps.

inside a neutral clump. The vast majority of the LyC photons that are emitted inside a cloud do not escape.

We thus need 14 parameters to completely characterize our models. ${ }^{5}$ Laursen et al. (2013) discuss plausible ranges for each parameter based on theoretical models and on observations of the ISM in the Milky Way, nearby dwarf galaxies, Ly $\alpha$ emitters (LAEs) and drop-out galaxies out to $z \sim 6$. Our fiducial model adopts the central value of the range quoted in Laursen et al. (2013) as "reasonable," with the exception of the outflow velocity $v_{\infty, \mathrm{cl}}$, for which Laursen et al. (2013) chose deliberately small values. Values for each parameter are listed in Table 1 in the Appendix. We assembled a library of 2500 spectra (using 10,000 escaped photons each). We drew each parameter uniformly ${ }^{6}$ from the range indicated in Table 1, which is loosely based on the "extreme" range in Laursen et al. (2013). This choice gives us a suite of empirical, simplified models of the multiphase ISM that span the wide range of physical conditions encountered in observed galaxies.

\section{RESULTS: CORRELATION BETWEEN $f_{\mathrm{esc}}^{\text {ion }}$ AND $f_{\mathrm{esc}}^{\mathrm{Ly} \alpha}$}

Figure 2 shows $f_{\text {esc }}^{\text {ion }}$ as a function of $f_{\text {esc }}^{\mathrm{Ly} \alpha}$. Each cross represents a Monte-Carlo radiative transfer simulation for one random realization of a clumpy ISM model. The color of the cross denotes $f_{\mathrm{cl}}$.

There are several points to conclude from this plot.

1. The 2500 models give rise to significant variation in $f_{\mathrm{esc}}^{\mathrm{Ly} \alpha}$ (which spans about three orders of magnitude) and $f_{\text {esc }}^{\text {ion }}$ (which spans about four orders of magnitude). Models that give rise to $f_{\mathrm{esc}}^{\mathrm{Ly} \alpha}>0.1-0.2$ would correspond to galaxies with relatively "strong" $\operatorname{Ly} \alpha$ emission, such as

\footnotetext{
5 Note that the parameters given here differ slightly from what we used in Gronke \& Dijkstra (2014). There, we ignored the filling of the ICM since we were interested in the (enhancement of) the Lyoescape fraction.

6 Note that $n_{\mathrm{H} \mathrm{IICM}}, n_{\mathrm{d} \text {, ICM }}, T_{\mathrm{ICM}}, T_{\mathrm{cl}}, Z_{\mathrm{cl}}$, and $\zeta_{\mathrm{Z}}$ were drawn uniformly in log-space.
}

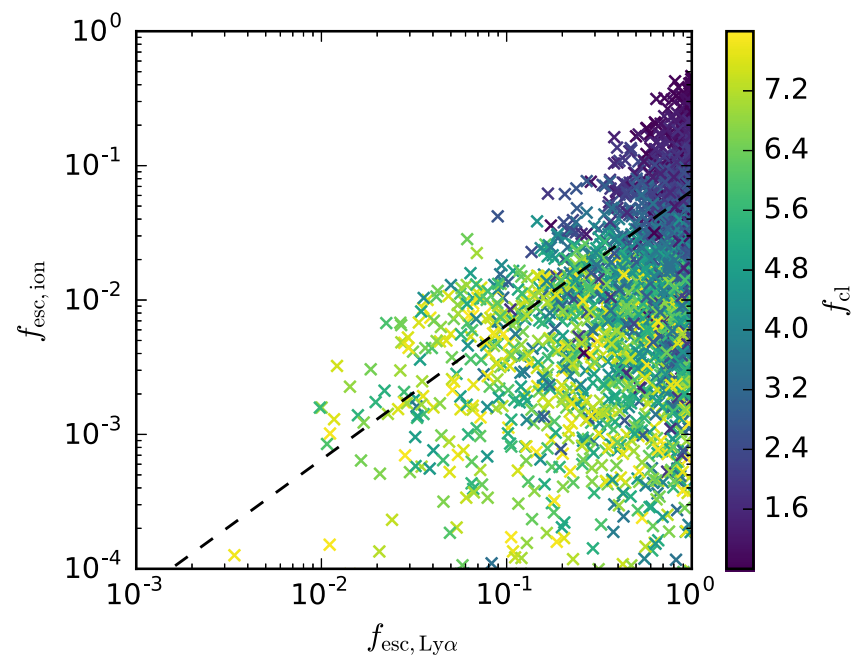

Figure 2. The escape fraction of ionizing photons, $f_{\mathrm{esc}}^{\text {ion }}$, as a function of the Ly $\alpha$ escape fraction, $f_{\mathrm{esc}}^{\mathrm{Ly} \alpha}$, for a suite of 2500 clumpy ISM models. Each cross represents the angle-averaged escape fraction for a complete Ly $\alpha$ Monte-Carlo radiative transfer calculation for one particular parameterization of the clumpy ISM model. The color of the crosses denotes the cloud covering factor $f_{\mathrm{cl}}$. This plot shows that there is a correlation between the two parameters: galaxies with low $f_{\text {esc }}^{\text {Ly } \alpha}$ have a low $f_{\text {esc }}^{\text {ion }}$, while galaxies with high $f_{\text {esc }}^{\text {Ly } \alpha}$ show a large spread in $f_{\mathrm{esc}}^{\text {ion }}$, driven strongly by $f_{\mathrm{cl}}$.

Ly $\alpha$ emitters. Our models therefore give rise to a population of "Ly $\alpha$ emitters" and weaker $\operatorname{Ly} \alpha$ sources such as drop-out galaxies with weak $\operatorname{Ly} \alpha$ emission. Our results also indicate that for fixed $f_{\mathrm{esc}}^{\mathrm{Ly} \alpha}$, the dispersion in $f_{\text {esc }}^{\text {ion }}$ can be large.

2. When $f_{\mathrm{esc}}^{\mathrm{Ly} \alpha}$ is small, then $f_{\mathrm{esc}}^{\text {ion }}$ is small. Ly $\alpha$ photons are destroyed most efficiently when they encounter, and scatter in, many different clumps. The number of scattering events (or "cloud interactions") scales as $\mathcal{N}_{\mathrm{cl}} \propto f_{\mathrm{cl}}^{2}\left(f_{\mathrm{cl}} \gg 1\right.$, Hansen \& Oh 2006). If $f_{\mathrm{cl}}$ is larger, then the Poisson probability of having clear sightlines becomes exponentially smaller: the Poisson probability that a sightline from $r=0$ intersects zero clumps is equal to ${ }^{7}$ $P\left(N_{\text {clump }}=0 \mid f_{\mathrm{cl}}, r=0\right)=\left(1-P_{\text {cloud }}\right) \exp \left(-f_{\mathrm{cl}}\right)$, where $1-P_{\text {cloud }}$ denotes the probability that the LyC photon was not emitted inside a cloud.

This result may make it difficult to explain the inferred $f_{\text {esc }}^{\text {ion }} \sim 0.1-0.2$ for a small subset Lyman break galaxies (LBGs; e.g., Iwata et al. 2009, Micheva et al. 2016). This apparent discrepancy can be alleviated in five ways. (i) Resonant scattering of Ly $\alpha$ off residual H I gas in the diffuse IGM can suppress the observed Ly $\alpha$ flux by an additional factor of 1.5-2.0 depending on redshift (e.g., Laursen et al. 2011), which should be applied to our predicted $f_{\mathrm{esc}}^{\mathrm{Ly} \alpha}$ when comparing to observations. (ii) The fraction of LBGs with claimed LyC detections is very small, which suggests that this population is rare and is not captured by our analysis in spite of our coverage of a broad range of physical conditions in the ISM. (iii) While LBGs generally have smaller $f_{\mathrm{esc}}^{\mathrm{Ly} \alpha}$ than LAEs, $f_{\mathrm{esc}}^{\mathrm{Ly} \alpha}$ appears to be correlated with the Ly $\alpha$ equivalent width (EW) (e.g.,

\footnotetext{
7 The expression for $P\left(N_{\text {clump }}=0 \mid f_{\mathrm{cl}}\right)$ that properly includes the Lya emissivity profile $\epsilon_{\mathrm{Ly} \alpha}(r)$ must take into account that the probability $P\left(N_{\text {clump }}=0 \mid f_{\mathrm{cl}}, r\right)$ depends on emission direction for $r \neq 0$. This makes the expression for $P\left(N_{\text {clump }}=0 \mid f_{\mathrm{cl}}\right)$ a bit more complicated but preserves the exponential dependence on $f_{\mathrm{cl}}$.
} 
Trainor et al. 2015, Micheva et al. 2016), which itself scales as $\mathrm{EW} \propto\left(1-f_{\mathrm{esc}}^{\mathrm{ion}}\right) f_{\mathrm{esc}}^{\mathrm{Ly} \alpha}($ see Section 4.4). This suggests that those LBGs that show LyC leakage may in fact have larger $f_{\mathrm{esc}}^{\mathrm{Ly} \alpha}$ than the LBG population as a whole. (iv) For very large $f_{\mathrm{esc}}^{\text {ion }}$, the production rate of Ly $\alpha$ decreases, which mimics a low $f_{\mathrm{esc}}^{\mathrm{Ly} \alpha}$ (see Section 4.4 for more discussion on this). (v) Each cross in Figure 2 represents an angle-average of the escape fractions for each of the 2500 models. The "apparent" $f_{\text {esc }}^{\text {ion }}$ can be larger along sightlines that do not intersect any clumps. The Ly $\alpha$ escape fraction can also be enhanced for these same sightlines, though scattering of Ly $\alpha$ photons suppresses the angular variation of $f_{\text {esc }}^{\text {Ly }}$ (see Gronke \& Dijkstra 2014). The angular variation of both escape fractions can be represented by replacing each cross in Figure 2 with a distribution that is elongated along the $f_{\mathrm{esc}}^{\text {ion }}$ direction, which may help explain why objects exist for which $f_{\mathrm{esc}}^{\text {ion }}$ is high while $f_{\mathrm{esc}}^{\mathrm{Ly} \alpha}$ is low.

3. The dispersion in $f_{\mathrm{esc}}^{\mathrm{ion}}$ at fixed $f_{\mathrm{esc}}^{\mathrm{Ly} \alpha}$ increases with $f_{\mathrm{esc}}^{\mathrm{Ly} \alpha}$. In other words, as we increase $f_{\mathrm{esc}}^{\mathrm{Ly} \alpha}$ the probability of having a large $f_{\mathrm{esc}}^{\text {ion }}$ increases. There are a number of ways to boost $f_{\mathrm{esc}}^{\mathrm{Ly} \alpha}$. These include reducing the dust content of the neutral clumps, increasing the outflow velocity, and reducing $f_{\mathrm{cl}}$. As mentioned above, reducing $f_{\mathrm{cl}}$ enhances the Poisson probability that there exist sightlines that do not intersect any clumps, which increases $f_{\mathrm{esc}}^{\text {ion }}$. Matthee et al. (2016a) recently found $f_{\mathrm{esc}}^{\text {ion }}>60 \%$ for eight $\mathrm{H} \alpha$ emitters (HAEs) out of a sample of 191. Two of these LyC-emitting HAEs have a high $f_{\mathrm{esc}}^{\mathrm{Ly} \alpha}$ (Matthee et al. 2016b). For the remaining six the $\operatorname{Ly} \alpha$ is not good enough (yet) to constrain $f_{\mathrm{esc}}^{\mathrm{Ly} \alpha}$.

4. The color coding of the crosses in Figure 2 shows clearly that models with high $f_{\mathrm{esc}}^{\text {ion }}$ are those with low $f_{\mathrm{cl}}$. This again reflects that a lower average number density of clouds from the center to the edge of the "galaxy" boosts the Poisson probability of having clean sightlines.

The strong dependence of $f_{\mathrm{esc}}^{\text {ion }}$ on $f_{\mathrm{cl}}$ is easily understood from analytic arguments. The simulations indicate that this result is not significantly affected by varying the other parameters. The black dashed line shows the best linear fit through the collection of data points. We stress that the purpose of this line is to illustrate that $f_{\mathrm{esc}}^{\mathrm{ion}}$ and $f_{\mathrm{esc}}^{\mathrm{Ly} \alpha}$ are correlated. The exact "best-fit" correlation depends on how the 14 model parameters were sampled: different probability density functions (pdfs) for the model parameters would likely yield a different best-fit correlation. This may help explain why our correlation differs quantitatively from that found by Yajima et al. (2014), who found few objects with high $f_{\mathrm{esc}}^{\mathrm{Ly} \alpha}$ and low $f_{\text {esc }}^{\text {ion }}$. This difference may also reflect that (i) the simulations do not resolve the multiphase ISM, and may therefore not properly capture the fact that $\operatorname{Ly} \alpha$ photons avoid destruction by dust by scattering off the surface of dense, neutral clumps that contain most of the dust, and (ii) that our model artificially enhances this surface scattering effect, by representing the multiphase ISM as a two-phase medium. We stress that the purpose of our calculations was not to derive the correct correlation, which would be overambitious, but rather to show that a correlation exists for reasonable parameters for the multiphase ISM. Finally, it is worth mentioning that the fact that both $f_{\mathrm{esc}}^{\text {ion }}$ and $f_{\mathrm{esc}}^{\mathrm{Ly} \alpha}$ are affected most strongly by $f_{\mathrm{cl}}$ implies that the precise structure of the clumps (i.e., the presence of a "cold neutral medium" inside the clumps) would introduce changes that are subdominant to those introduced by $f_{\mathrm{cl}}$.

\section{DISCUSSION}

\subsection{The $M_{\mathrm{Uv}}$ Dependence of $f_{\mathrm{esc}}^{\mathrm{Ly}}$}

The "Ly $\alpha$ fraction" denotes the fraction of galaxies that have a Ly $\alpha$ emission line stronger than some threshold EW. Observations indicate that the Ly $\alpha$ fraction increases with $M_{\mathrm{UV}}$ (e.g., Stark et al. 2010; Pentericci et al. 2011, 2014; Caruana et al. 2012, 2014; Ono et al. 2012; Schenker et al. 2012). Gronke et al. (2015b) combined observations of the UV luminosity function (UV LF) with current constraints on the UV dependence of the $\operatorname{Ly} \alpha$ fraction, and predicted that Ly $\alpha$ LFs should have steeper faint ends than the UV LFs. Specifically, if we denote the faint-end slope of the Ly $\alpha$ LF by $\alpha_{\mathrm{Ly} \alpha}$, then $\alpha_{\mathrm{Ly} \alpha}=\alpha_{\mathrm{UV}}-x$ where $x \sim 0.2-0.4$ (see Figure 2 of Gronke et al. 2015b). Recent measurements of the faint-end slope of the luminosity functions of $\operatorname{Ly} \alpha$ emitters at $z=5.7$ indicate that $\alpha_{\mathrm{Ly} \alpha} \sim-2.2 \pm 0.2$ (Dressler et al. 2015), and that $\alpha_{\text {Ly } \alpha} \sim-1.75 \pm 0.1$ at $z \sim 2$ (Konno et al. 2016). These measurements agree well ${ }^{8}$ with prediction using constraints on Ly $\alpha$ fraction, and provide independent confirmation that more Ly $\alpha$ radiation emerges per "unit" UV flux density toward lower UV luminosities.

This enhanced emergence of $\operatorname{Ly} \alpha$ flux from UV-faint galaxies implies that (i) the $\mathrm{Ly} \alpha$ production rate increases and/or (ii) $f_{\text {esc }}^{\text {Ly } \alpha}$ increases toward lower UV luminosities. Recent work has shown that at $z \sim 4$ the production efficiency of ionizing photons, $\xi_{\text {ion }}$ (Robertson et al. 2013), appears to be independent of $M_{\mathrm{UV}}$ in the range $-21<M_{\mathrm{UV}}<-19$ at $z \sim 4-5$ (see Figure 1 of Bouwens et al. 2016, which also shows that there is still a large scatter). The Ly $\alpha$ production efficiency should then also not depend on $M_{\mathrm{UV}}$, because the production of $\operatorname{Ly} \alpha$ is directly tied to the production of ionizing photons. In contrast, over this same range in $M_{\mathrm{UV}}$, the Ly $\alpha$ fraction rises rapidly (see Figure 13 of Stark et al. 2010). This suggests that the enhanced visibility of $\operatorname{Ly} \alpha$ flux is mostly driven by an enhanced escape fraction, and provides the basis for our statement that there is observational support that $f_{\mathrm{esc}}^{\mathrm{Ly} \alpha}$ increases toward lower UV luminosities (or toward higher $\left.M_{\mathrm{UV}}\right)$. Trainor et al. (2015) note that in LAEs with $\mathrm{H} \alpha$ detections, the inferred $f_{\mathrm{esc}}^{\mathrm{Ly} \alpha}$ correlates significantly with Ly $\alpha$ $\mathrm{EW}$, which provides independent confirmation that $\mathrm{Ly} \alpha \mathrm{EW}$ is an indicator of $\mathrm{Ly} \alpha$ escape.

Oyarzún et al. (2016) recently found that the pdf of Ly $\alpha \mathrm{EW}$, and therefore the Ly $\alpha$ fraction, depends on stellar mass, $M_{*}$. This supports the idea that $f_{\mathrm{esc}}^{\mathrm{Ly} \alpha}$ increases toward lower $M_{*}^{*}$. Our finding of a correlation between $f_{\mathrm{esc}}^{\mathrm{Ly} \alpha}$ and $f_{\mathrm{esc}}^{\text {ion }}$ then implies that $f_{\mathrm{esc}}^{\text {ion }}$ also increases toward lower $M_{*}$. Faisst (2016) independently came to this conclusion by combining the observed correlation between $f_{\text {esc }}^{\text {ion }}$ and the line ratio [O III] $\lambda 5007 /[\mathrm{O} \mathrm{II}] \lambda 3727$, and the (anti)correlation of this line ratio with $M_{*}$ inferred from local high- $z$ analogues. We stress that we focus on the $M_{\mathrm{UV}}$ dependence of $f_{\text {esc }}^{\text {ion }}$ because this allows us

\footnotetext{
8 Gronke et al. (2015b) only predicted Ly $\alpha$ LFs at $z \geqslant 3$. We extrapolated their predictions for $\alpha_{\mathrm{Ly} \alpha}$ to $z \sim 2$. This same extrapolation would translate to a faint-end slope of the UV luminosity function at $z \sim 2.3$ that is $\alpha_{\mathrm{UV}} \sim-1.5$, which agrees with recent determinations (see Figure 10 of Parsa et al. 2016), though not all (see, e.g., Reddy \& Steidel 2009, who found a steeper $\left.\alpha_{\mathrm{UV}}=-1.73 \pm 0.07\right)$.
} 


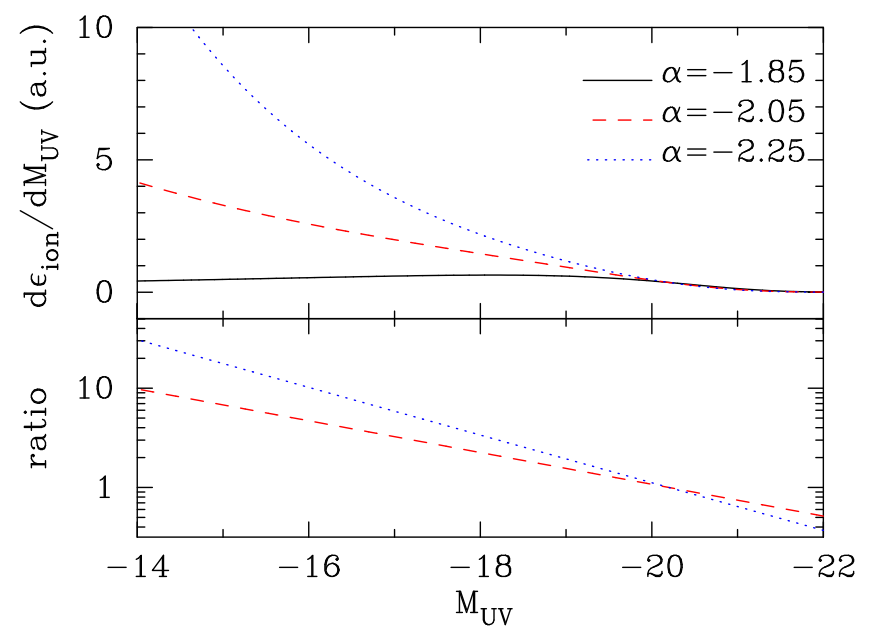

Figure 3. An increase in $f_{\mathrm{esc}}^{\text {ion }}$ toward lower UV luminosities gives rise to a steepening of the LyC luminosity function, which can be mimicked with a steeper UV LF and a constant $f_{\text {esc }}^{\text {ion }}$. The top panel shows the relative contribution $d \epsilon_{\mathrm{ion}} / d M_{\mathrm{UV}}$ (in arbitrary units) to the ionizing volume emissivity $\epsilon_{\text {ion }}$ at $z=6$ by galaxies with $M_{\mathrm{UV}} \pm d M_{\mathrm{UV}} / 2$ for the measured $\alpha_{\mathrm{UV}}=-1.85$ (black solid line), and steeper LFs with $\alpha_{\mathrm{UV}}=-2.25$ (blue dotted line) and $\alpha_{\mathrm{UV}}=-2.05$ (red dashed line). While we cannot predict (yet) which $\alpha_{\mathrm{UV}}$ mimics the true $M_{\mathrm{UV}}$ dependence of $f_{\mathrm{esc}}^{\text {ion }}$, this plot visually illustrates the enhanced contribution of UV-faint galaxies to cosmic reionization. The lower panel shows the ratio between the models in the top panel and the fiducial model (shown as the black solid line).

to directly connect our results to the UV LF of continuumselected galaxies, which is routinely used to quantify the $\mathrm{LyC}$ volume emissivity of galaxies during cosmic reionization.

\subsection{Implications for Reionization}

One of the main open questions in reionization is whether galaxies provided enough photons to reionize the universe, and if so, which galaxies provided the dominant contribution to the ionizing background that drove reionization. These questions are commonly addressed by extrapolating the faint end of the (non-ionizing) UV LF of drop-out galaxies to some minimum UV luminosity (corresponding to a maximum $M_{\mathrm{UV}}^{\mathrm{lim}}$ ), and then seeing whether these galaxies provided enough photons either to reionize the universe or to keep it ionized (e.g., Wilkins et al. 2011; Finkelstein et al. 2012a; Kuhlen \& FaucherGiguère 2012; Shull et al. 2012; Robertson et al. 2013). This approach introduces two parameters related to the UV LF: (i) its faint-end slope $\left(\alpha_{\mathrm{UV}}\right)$ and (ii) its minimum cutoff luminosity $\left(M_{\mathrm{UV}}^{\lim }\right)$. For a fixed set of parameters $\left(\alpha_{\mathrm{UV}}, M_{\mathrm{UV}}^{\lim }\right)$, the question of whether galaxies reionized the universe then translates to a constraint on $f_{\mathrm{esc}}^{\text {ion }}$. This constraint on $f_{\mathrm{esc}}^{\text {ion }}$ represents a (weighted) average over the full population of UV-emitting galaxies. There have been numerous theoretical efforts to model the faint-end slope of the UV LF and where it may flatten (e.g., Jaacks et al. 2013; Mason et al. 2015; O'Shea et al. 2015; Liu et al. 2016).

If the escape of $\mathrm{Ly} \alpha$ and $\mathrm{LyC}$ are correlated, then we also expect $f_{\text {esc }}^{\text {ion }}$ to increase toward lower UV luminosities. Just as in the case for $\mathrm{Ly} \alpha$, if we were to plot the $\mathrm{LyC}$ luminosity function (i.e., the number density of galaxies as a function of LyC luminosity), it would be steeper than the UV luminosity function. This steepening can be mimicked by a model in which $f_{\mathrm{esc}}^{\text {ion }}$ does not depend on $M_{\mathrm{UV}}$, and in which the faint-end slope of the UV luminosity function is made steeper. Figure 3 visually illustrates the impact of this steepening, and the top panel shows the relative contribution $d \epsilon_{\mathrm{ion}} / d M_{\mathrm{UV}}$ to the ionizing volume emissivity $\epsilon_{\text {ion }}$ by galaxies in the range $M_{\mathrm{UV}} \pm d M_{\mathrm{UV}} / 2$. The black solid line shows $d \epsilon_{\mathrm{ion}} / d M_{\mathrm{UV}}$ for the "standard" Schechter function parameters at $z=6,\left(\alpha_{\mathrm{UV}}\right.$, $\left.M_{*}\right)=(-1.85,-20.2)$ (using the fitting formula from Bouwens et al. 2015). For the blue dotted line [red dashed line] we increased $\alpha_{\mathrm{UV}} \rightarrow-2.25$, which represents the steepening relevant for the Ly $\alpha$ LF $\left[\alpha_{\mathrm{UV}} \rightarrow-2.05\right.$, which represents an intermediate case]. While we do not know which $\alpha_{\mathrm{UV}}$ mimics the correct $M_{\mathrm{UV}}$ dependence of $f_{\mathrm{esc}}^{\text {ion }}$, the plot does illustrate the possible enhanced contribution of UV-faint galaxies to cosmic reionization. ${ }^{9}$ The enhancement is illustrated in the lower panel of Figure 3, which shows the ratio of the models shown in the top panel. This plot shows that in a model with $\alpha_{\mathrm{UV}}=-2.25$ galaxies with $M_{\mathrm{UV}} \sim-16$ contribute $>10$ times more to the total rate of ionizing photon production than when $\alpha=-1.85$. This model extends down to $M_{\mathrm{UV}}=-14$, which corresponds (roughly) to the limit to which the UV LF has been constrained to be a power law (see, e.g., Alavi et al. 2014; Livermore et al. 2016; Parsa et al. 2016; also see O'Shea et al. 2015 and Liu et al. 2016 for theoretical arguments why the UV LF may flatten at $M_{\mathrm{UV}}>-14$ ).

Finally, Ly $\alpha$ escape in clumpy ISM models-which were introduced to reflect the multiphase nature of the ISM-is most strongly regulated by covering factor, and to a lesser extent by other parameters such as the dust content (Gronke \& Dijkstra 2016). Star-forming galaxies are known to become bluer toward higher redshift, which is taken as evidence that they get less dusty toward higher redshifts (e.g., Bouwens et al. 2012; Finkelstein et al. 2012b). Our conclusions would break down if Ly $\alpha$ escape were driven entirely by the changing dust content of an otherwise identical scattering medium. In this case, however, we would expect both the width and (possibly) velocity shift of the Ly $\alpha$ line to increase with $M_{\mathrm{UV}}$, because Ly $\alpha$ scattering causes photons to diffuse in frequency space and to broaden the Ly $\alpha$ spectral line shape. If only dust were regulating Ly $\alpha$ escape, then dust would suppress this frequency diffusion and cause lines to be narrower (see, e.g., Figure 8 of Laursen et al. 2009). The evolution in the width and shift of the Ly $\alpha$ line predicted by the "pure dust" scenario is not consistent with observations that indicate that $\operatorname{Ly} \alpha$ spectra of $\operatorname{Ly} \alpha$ emitting galaxies tend, if anything, to get narrower: Konno et al. (2016) have shown that shell-model fits to observed Ly $\alpha$ line profiles favor increasingly low H I column densities toward higher $z$ for otherwise identical shell-model parameters. The reduced $\mathrm{H} \mathrm{I}$ column density introduces less frequency diffusion and makes Ly $\alpha$ line profiles narrower. ${ }^{10}$ In addition, there is observational support that the covering factor of low-ionization metal lines decreases with $z$ (e.g., Jones et al. 2013). If these metals trace cold, neutral gas, then this supports the notion that Ly $\alpha$ escape increases toward higher redshift (at least partly) because of the evolution in the covering factor of neutral gas.

\footnotetext{
9 Decreasing $\alpha$ reduces the contribution of UV-bright galaxies, i.e., $M_{\mathrm{UV}}<M_{*}$, to $d \epsilon_{\mathrm{ion}} / d M_{\mathrm{UV}}$ because reducing $\alpha$ also affects the bright end of the luminosity function.

${ }^{10}$ Also, the velocity offset of the peak flux density of the Ly $\alpha$ spectral line shape decreases toward UV-fainter galaxies (e.g., Erb et al. 2014; Song et al. 2014).
} 


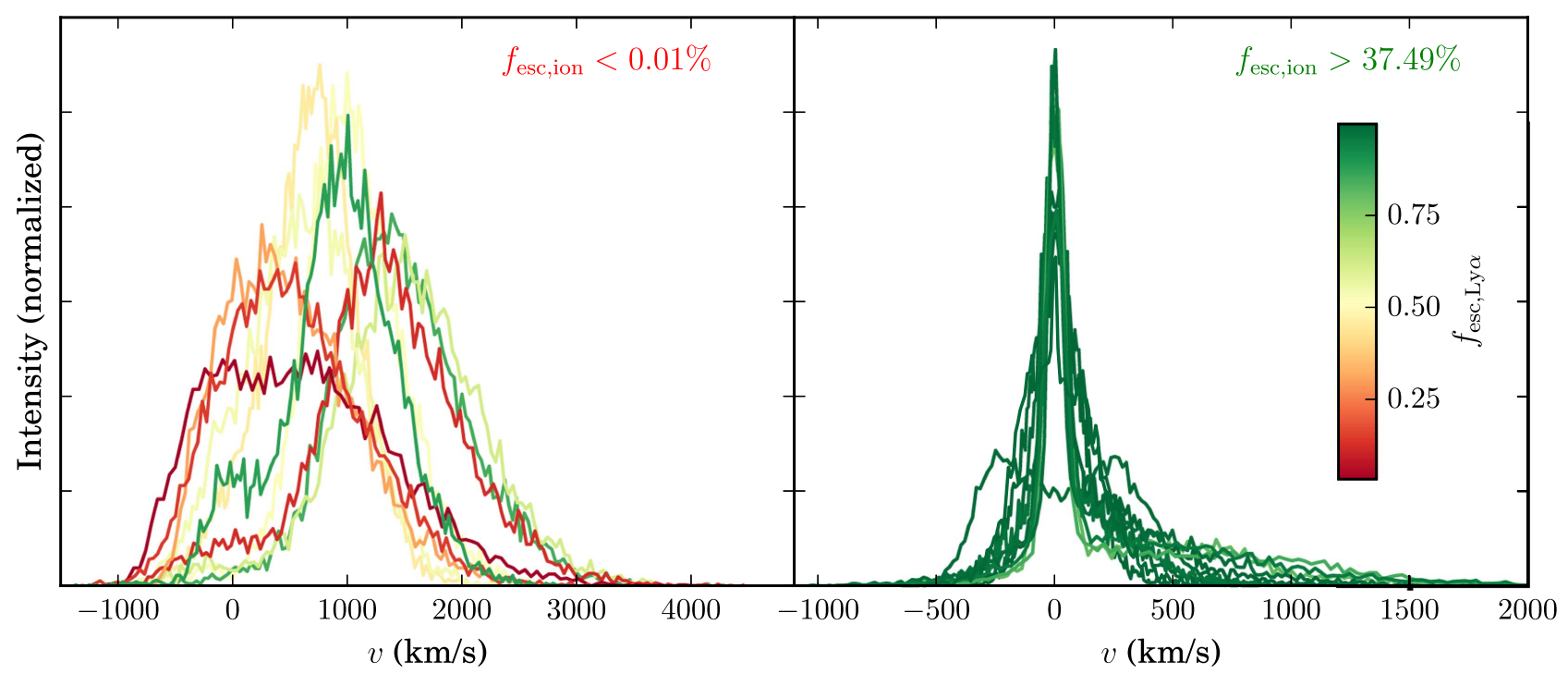

Figure 4. Left panel: Ly $\alpha$ spectra emerging from 25 models with the lowest $f_{\mathrm{esc}}^{\text {ion }}<10^{-4}$. Right panel: Ly $\alpha$ spectra emerging from the 25 models with the highest $f_{\text {esc }}^{\text {ion }}>0.37$. This figure shows that for models with low $f_{\text {esc }}^{\text {ion }}$ Ly $\alpha$ spectra are redshifted, asymmetric, and broad. The width and velocity offset of the models with the lowest $f_{\text {esc }}^{\text {ion }}$ are larger than what has been observed, which is likely because of the simplified representation of the multiphase ISM (see text). Although models with high $f_{\mathrm{esc}}^{\text {ios }}$ exhibit a variety of spectral line shapes, their spectra are generally narrower and more symmetric.

\subsection{Connection of $f_{\mathrm{esc}}^{\mathrm{ion}}$ to the Lya Spectrum}

Figure 2 showed that $f_{\mathrm{esc}}^{\text {ion }}$ depends sensitively on $f_{\mathrm{cl}}$, which is due to the exponential dependence on $f_{\mathrm{cl}}$ of the Poisson probability of having sightlines with no clumps. The parameter $f_{\mathrm{cl}}$ is known to play a key role in Ly $\alpha$ transfer through clumpy media (Hansen \& Oh 2006). We have demonstrated that $f_{\mathrm{cl}}$ is one of the 14 parameters of the clumpy models that most strongly affects the emerging Ly $\alpha$ spectrum (Gronke \& Dijkstra 2016). This implies immediately that $f_{\text {esc }}^{\text {ion }}$ should be closely correlated with spectral features of the Ly $\alpha$ line.

Figure 4 compares Ly $\alpha$ spectra for 25 models with the highest $f_{\text {esc }}^{\text {ion }}>0.37$ (right panel) to 25 models with the lowest $f_{\text {esc }}^{\text {ion }}<10^{-4}$ (left panel). These two panels illustrate clearly that a high $f_{\text {esc }}^{\text {ion }}$ corresponds to having narrower, more symmetric Ly $\alpha$ lines. Models that have the highest $f_{\text {esc }}^{\text {ion }}$ show a variety in their spectra. We caution that the width and velocity offset of the models with the lowest $f_{\text {esc }}^{\text {ion }}$ are larger than what has been observed. This is likely an artefact of the models: models with the lowest $f_{\text {esc }}^{\text {ion }}$ have the highest $f_{\mathrm{cl}} \sim 8$. Ly $\alpha$ photons typically scatter off $\sim f_{\mathrm{CL}}^{2}$ separate clouds before escaping (e.g., Hansen $\&$ Oh 2006), and each "cloud-interaction" can impart a noticeable Doppler boost to the Ly $\alpha$ photon, which broadens the Ly $\alpha$ spectral line.

The connection between the Ly $\alpha$ spectral shape and the escape of ionizing photons was pointed out previously by Behrens et al. (2014, in the context of modified shell models) and Verhamme et al. (2015, in the context of shell models). In these models, LyC escape translated to (i) significant $\mathrm{Ly} \alpha$ flux at systemic velocity and/or (ii) a small peak separation $\left(\Delta v<300 \mathrm{~km} \mathrm{~s}^{-1}\right)$. In multiphase models, it is not possible to point out features in the spectrum that guarantee a $\mathrm{LyC}$ detection, partly because of the larger variety in the spectra associated with models that have larger $f_{\text {esc }}^{\text {ion }}$. In addition, in clumpy models Ly $\alpha$ photons can escape after scattering off a single gas cloud, and close to the frequency at which they were initially emitted (also see Hansen \& Oh 2006; Laursen et al.
2013). Moreover, while narrow Ly $\alpha$ lines that are symmetric around the systemic velocity of the host galaxy translate to a higher probability of being a LyC-emitting galaxy, LyC escape is highly anisotropic (Ly $\alpha$ escape less so, see Gronke \& Dijkstra 2014), which further complicates the making of robust predictions as to whether we can observe LyC flux from a galaxy or not. However, anisotropic escape of LyC photons similarly affects other promising indicators of LyC leakage ${ }^{11}$ such as the line ratio [O III] $\lambda 5007 /[\mathrm{O}$ II $] \lambda 3727$ (Jaskot \& Oey 2013; Nakajima \& Ouchi 2014).

The low-redshift "Lyman break analogue" (LBA, Heckman et al. 2011; Borthakur et al. 2014) and "green pea galaxy" (Henry et al. 2015; Izotov et al. 2016; Yang et al. 2016), with reported detections of LyC escape, had unusual Ly $\alpha$ spectra in the sense that the spectra contained significant flux blueward of Ly $\alpha$ resonance. These spectra were different than those shown in Figure 4 in that they had deep "absorption" troughs separating the blue and red peaks, which are absent from the spectra in Figure 4. The absence of these absorption troughs from the theoretical spectra may reflect the lack of trace amounts of residual HI (possibly in the circumgalactic medium) at systemic velocity (see Gronke \& Dijkstra 2016). In any case, the presence of flux blueward of the Ly $\alpha$ resonance indicates that the lines are more symmetric around the $\operatorname{Ly} \alpha$ resonance than is common.

\subsection{Suppressed Lya Production for Large $f_{\mathrm{esc}}^{\text {ion }}$}

The Ly $\alpha$ production rate scales as $\propto\left(1-f_{\mathrm{esc}}^{\text {ion }}\right)$. The total Ly $\alpha$ flux that we receive from a distant galaxy and the EW of the line both scale as $\propto\left(1-f_{\mathrm{esc}}^{\text {ion }}\right) f_{\mathrm{esc}}^{\mathrm{Ly} \alpha}$.

Figure 5 shows $\log f_{\text {esc }}^{\text {ion }}$ as a function of $\log \left[f_{\mathrm{esc}}^{\mathrm{Ly} \alpha}\left(1-f_{\mathrm{esc}}^{\text {ion }}\right)\right]$. The turnover at high $f_{\text {esc }}^{\text {ion }}$ and high $f_{\text {esc }}^{\text {Ly } \alpha}$ reflects the fact that the quantity $f_{\mathrm{esc}}^{\mathrm{Ly} \alpha}\left(1-f_{\mathrm{esc}}^{\mathrm{ion}}\right)$ cannot exceed $\left(1-f_{\mathrm{esc}}^{\mathrm{ion}}\right)$ (which is

\footnotetext{
11 On the other hand, LyC escape enhances the ionizing radiation field in close proximity to star-forming galaxies, which can increase the surface brightness in fluorescent Ly $\alpha$ and $\mathrm{H} \alpha$ emission (see Mas-Ribas \& Dijkstra 2016).
} 


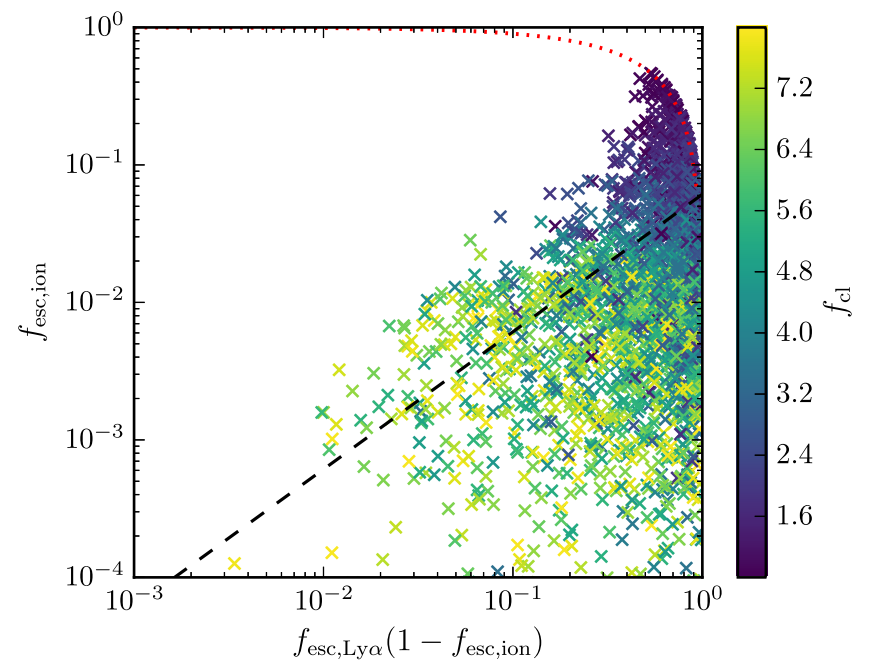

Figure 5. This plot shows $f_{\mathrm{esc}}^{\text {ion }}$ as a function of the "apparent" Ly $\alpha$ escape fraction, $f_{\mathrm{esc}}^{\mathrm{Ly} \alpha}\left(1-f_{\mathrm{esc}}^{\mathrm{ion}}\right)$, which reflects the fact that the production rate of Ly $\alpha$ photons scales as $\propto\left(1-f_{\mathrm{esc}}^{\text {ion }}\right)$. The red dotted line shows the maximum apparent escape fraction $\left(1-f_{\mathrm{esc}}^{\text {ion }}\right)$. Large $f_{\mathrm{esc}}^{\text {ion }}$ thus also suppresses the observed Ly $\alpha$ flux, mimicking a reduction in $f_{\mathrm{esc}}^{\mathrm{Ly} \alpha}$. This Figure illustrates that there exists a maximum average $f_{\mathrm{esc}}^{\mathrm{Ly} \alpha}\left(1-f_{\mathrm{esc}}^{\mathrm{ion}}\right)$ at some $f_{\mathrm{esc}}^{\text {ion }} \equiv f_{\mathrm{esc}, \max }^{\text {ion }} \sim 0.1-0.5$ (see text).

indicated by the red dotted line). At fixed $f_{\mathrm{esc}}^{\text {ion }}$ there exists a distribution of $f_{\mathrm{esc}}^{\mathrm{Ly} \alpha}\left(1-f_{\mathrm{esc}}^{\mathrm{ion}}\right)$ that reflects the dispersion in $f_{\text {esc }}^{\text {Ly } \alpha}$. The average of this distribution peaks at some maximum $f_{\text {esc,max }}^{\text {ion }}$ (also see Dijkstra et al. 2014). The value of $f_{\text {esc,max }}^{\text {ion }}$ is model-dependent, and even in the context of our model it depends on how we sampled our 14 parameters. It nevertheless seems reasonable to assume that $f_{\mathrm{esc} \text {,max }}^{\mathrm{ion}} \sim 0.1-0.5$. For large $f_{\text {esc }}^{\text {ion }}>f_{\text {esc,max }}^{\text {ion }}$ the Ly $\alpha$ luminosity drops again, which mimics a reduction in $f_{\mathrm{esc}}^{\mathrm{Ly} \alpha}$. We expect this "apparent" reduction in the escape fraction to translate to a reduction in the $\operatorname{Ly} \alpha$ fraction and/or a flattening of the Ly $\alpha$ luminosity function at low Ly $\alpha$ luminosities. $^{12}$ No evidence for either this drop or this flattening exists at $z \sim 6$ in current data (though it may be present at $z \sim 6.5$, see Figure 7 of Matthee et al. 2015), which implies that this effect is not important in current observations at $z \sim 6$. More quantitatively, Dressler et al. (2015) infer a steep faint-end slope of the LAE LF down to $L_{\alpha}<10^{42} \mathrm{erg} \mathrm{s}^{-1}$. Gronke et al. (2015b) show that Ly $\alpha$ luminosity of $L_{\alpha} \sim 10^{42} \mathrm{erg} \mathrm{s}^{-1}$ probes galaxies with $M_{\mathrm{UV}} \sim-18 \pm 1$ (see their Figure 3 ). This therefore implies that $f_{\mathrm{esc}}^{\text {ion }}<f_{\mathrm{esc}, \max }^{\text {ion }}$, and therefore that this effect is not important, down to $M_{\mathrm{UV}} \sim-18 \pm 1$.

At $z>6$ there is observational evidence for a reduction in the Ly $\alpha$ flux from star-forming galaxies compared to expectations based on extrapolations from lower redshift observations (see, e.g., Dijkstra 2014 and references therein). There are indications that this reduction is more severe for UV-faint galaxies (e.g., Ono et al. 2012; Pentericci et al. 2014), which is commonly interpreted as a signature of inhomogeneous reionization, but might also reflect the fact that $f_{\text {esc }}^{\text {ion }} \rightarrow f_{\text {esc,max }}^{\text {ion }}$ in UV-faint galaxies at $z \sim 7$ (also see Dijkstra et al. 2014). It is theoretically possible to distinguish between

\footnotetext{
12 Although a flattening has possibly been detected at $z \sim 3$ by Rauch et al. (2008) at $L_{\alpha} \sim 10^{41} \mathrm{erg} \mathrm{s}^{-1}$, which would probe galaxies with $M_{\mathrm{UV}} \sim-15 \pm 1$ (see Gronke et al. 2015a for a discussion).
}

these two scenarios: (i) reionization leaves a unique signature on the angular clustering of Ly $\alpha$ emitters (McQuinn et al. 2007; Mesinger \& Furlanetto 2008; Jensen et al. 2013; Sobacchi \& Mesinger 2015), which can be measured with Subaru's Hyper Suprime-Cam ${ }^{13}$ (see, e.g., Jensen et al. 2014; Sobacchi \& Mesinger 2015); (ii) if Ly $\alpha$ disappears as a result of $f_{\mathrm{esc}}^{\text {ion }}$ becoming large, then we should see a similar decrease in the line strength of other non-resonant nebular lines such as $\mathrm{H} \alpha$ (and $\mathrm{H} \beta$ ), something that can be tested with the James Webb Space Telescope ${ }^{14}$ (Gardner et al. 2006).

Redshift $z \sim 6$ is particularly interesting because reionization likely had little impact on the observed Ly $\alpha$ flux from galaxies. Should future data reveal a flattening in the Ly $\alpha$ LF at low Ly $\alpha$ luminosities and/or a reduction in the $\operatorname{Ly} \alpha$ fraction at lower UV luminosities, then this may provide a valuable constraint on $f_{\text {esc }}^{\text {ion }}$ at this redshift. In addition, understanding whether $f_{\text {esc }}^{\text {ion }}$ introduces a flattening in the $\operatorname{Ly} \alpha \mathrm{LF}$ at low $\mathrm{Ly} \alpha$ luminosities and/or a drop in the Ly $\alpha$ fraction at faint UV luminosities would help us to better constrain the role that reionization plays in suppressing the Ly $\alpha$ flux from galaxies at $z>6$.

\subsection{Impact of Delayed Lya Escape due to Trapping}

The escape fraction of LyC photons from a galaxy can vary significantly on timescales of $\sim 10 \mathrm{Myr}$ (Kimm \& Cen 2014; Ma et al. 2015), which corresponds approximately to the lifetime of massive stars. Trapping of Ly $\alpha$ photons by $\mathrm{H}$ I gas can introduce a lag in the escape of $\mathrm{Ly} \alpha$ and $\mathrm{LyC}$ photons (Yajima \& Li 2014): Ly $\alpha$ photons scatter inside H I gas when $f_{\text {esc }}^{\text {ion }} \ll 1$ but are "released" efficiently when channels of low column density temporarily open up and allow LyC photons to escape. Temporal variations in $f_{\mathrm{esc}}^{\mathrm{ion}}$ and delayed escape of Ly $\alpha$ have only a minor, positive, impact on our results by slightly more tightly coupling $f_{\mathrm{esc}}^{\mathrm{Ly} \alpha}$ and $f_{\mathrm{esc}}^{\mathrm{ion}}$, as we explain below.

Trapping of Ly $\alpha$ photons is limited to timescales $t_{\text {trap }} \ll 10$ Myr, because the typical $\operatorname{Ly} \alpha$ trapping time is equal to $t_{\text {trap }}=\left|x_{\mathrm{p}}\right| t_{\text {cross }}$, where $t_{\text {cross }} \equiv R / c$ denotes the time it takes radiation to escape in the absence of scattering, and $\left|x_{\mathrm{p}}\right| \approx 12\left(N_{\mathrm{H}} / 10^{20} \mathrm{~cm}^{-2}\right)^{1 / 3}\left(T / 10^{4} \mathrm{~K}\right)^{1 / 6}$ for a static, uniform, spherical gas cloud of radius $R$ with an $\mathrm{HI}$ column density $N_{\mathrm{H} \text { I }}$ and temperature $T$ (Adams 1975). In reality, this estimate provides a strict upper limit to the delay time: velocity gradients and density inhomogeneities reduce $t_{\text {trap }}$ (Bonilha et al. 1979; Dijkstra \& Loeb 2008). Laursen et al. (2013) evaluated the typical trapping time for Ly $\alpha$ in clumpy media considered here to be $t_{\text {trap }} \sim 2 \times 10^{4} \mathrm{yr}$. Trapping of Ly $\alpha$ photons is therefore unlikely to introduce a lag between the escape of $\mathrm{Ly} \alpha$ and $\mathrm{LyC}$ photons at a level where it has observable consequences. Moreover, if anything, this effect would serve to couple $\mathrm{Ly} \alpha$ and LyC escape more tightly, because LyC escape would be accompanied by the escape of Ly $\alpha$ photons that were trapped inside the H I gas.

\section{CONCLUSIONS}

The escape fraction of ionizing photons, $f_{\mathrm{esc}}^{\text {ion }}$, represents one of the great unknowns in our understanding of cosmic reionization. Observational constraints on $f_{\text {esc }}^{\text {ion }}$ are still weak, and theoretical predictions remain incomplete owing to the challenging nature of the calculations. We have computed the

\footnotetext{
13 http://www.naoj.org/Projects/HSC/

14 http://www.jwst.nasa.gov/
} 
correlation between the escape fractions of $\operatorname{Ly} \alpha\left(f_{\mathrm{esc}}^{\mathrm{Ly} \alpha}\right)$ and ionizing (LyC) radiation $\left(f_{\mathrm{esc}}^{\text {ion }}\right)$ by performing Monte-Carlo simulations of $\mathrm{Ly} \alpha$ radiative transfer through a suite of 2500 models of dusty, clumpy interstellar media. This represents a "top-down" (empirical) approach to modeling LyC and Lya transfer through realistic, multiphase interstellar media, and complements the previous "bottom-up" (ab initio) approach by Yajima et al. (2014), who used hydrodynamical simulations to generate models of the ISM. Our main results are:

1. We find that $f_{\mathrm{esc}}^{\mathrm{ion}}$ and $f_{\mathrm{esc}}^{\mathrm{Ly} \alpha}$ are correlated. The dispersion in $f_{\text {esc }}^{\text {ion }}$ at fixed $f_{\text {esc }}^{\mathrm{Ly} \alpha}$ increases toward larger $f_{\mathrm{esc}}^{\mathrm{Ly} \alpha}$ : galaxies with low $f_{\mathrm{esc}}^{\mathrm{Ly} \alpha}$ have a low $f_{\mathrm{esc}}^{\text {ion }}$, while galaxies with high $f_{\mathrm{esc}}^{\mathrm{Ly} \alpha}$ show a large spread in $f_{\mathrm{esc}}^{\text {ion }}$ (see Figure 2). The dispersion in $f_{\mathrm{esc}}^{\text {ion }}$ is driven by the dispersion in $f_{\mathrm{cl}}$, which measures the cloud covering factor. Our results agree qualitatively with those obtained by Yajima et al. (2014), who also found a positive correlation, but quantitatively some differences remain, which reflects the fact that neither approach has converged yet (see the discussion in Section 2).

While predictions of both $f_{\mathrm{esc}}^{\text {ion }}$ and $f_{\mathrm{esc}}^{\mathrm{Ly} \alpha}$ are still highly uncertain, the existence of a correlation between the two quantities can be predicted more robustly, which is underlined by the fact that two different, independent approaches confirm the existence of this correlation. The $f_{\text {esc }}^{\mathrm{Ly} \alpha}-f_{\text {esc }}^{\text {ion }}$ correlation reflects the fact that the escape of ionizing photons requires that sightlines exist that contain low column densities of atomic hydrogen, i.e., $N_{\mathrm{H} \mathrm{I}}<1 /$ $\sigma_{\text {ion }} \approx 10^{17} \mathrm{~cm}^{-2}$. These same paths of low column density provide escape routes for Ly $\alpha$ photons (also see Behrens et al. 2014; Verhamme et al. 2015). At a deeper level, the escape of Ly $\alpha$ is facilitated by outflows, which may also create holes of low column density out of galaxies, which in turn permit LyC photons to escape.

2. We argued that the positive correlation between $f_{\mathrm{esc}}^{\text {ion }}$ and $f_{\text {esc }}^{\text {Ly } \alpha}$ is directly relevant for studies of cosmic reionization, because there is increasing observational support from both continuum- and line-selected galaxies that Ly $\alpha$ escapes more easily from UV-faint galaxies (see Section 4.1). The correlation between $f_{\mathrm{esc}}^{\mathrm{ion}}$ and $f_{\mathrm{esc}}^{\mathrm{Ly} \alpha}$ then implies that ionizing photons also escape more easily from UV-faint galaxies at this redshift. This implies that UV-faint galaxies contribute more to the volume emissivity of ionizing photons than is implied by the faint-end slope of the UV luminosity function (Section 4.2). These conclusions may be invalidated if the escape of Ly $\alpha$ is regulated purely by dust. However, we argued in Section 4.2 that observations do not support this picture.

3. Because the "apparent" Ly $\alpha$ escape fraction, $f_{\mathrm{esc}}^{\mathrm{Ly} \alpha}(1-$ $f_{\text {esc }}^{\text {ion }}$, reaches a maximum value for $f_{\text {esc }}^{\text {ion }}=f_{\text {esc,max }}^{\text {ion }} \sim$ $0.1-0.5$ (see Section 4.4), we expect a drop in the Ly $\alpha$ fraction at lower UV luminosities and/or a flattening of the $\operatorname{Ly} \alpha \mathrm{LF}$ at lower Ly $\alpha$ luminosities, if $f_{\mathrm{esc}}^{\text {ion }}$ continues to rise monotonically. This has not been observed yet at $z \sim 6$ (but possibly at $z \sim 6.5$, see Matthee et al. 2015), which implies that $f_{\mathrm{esc}}^{\text {ion }}<f_{\mathrm{esc} \text {,max }}^{\text {ion }}$ in galaxies with $M_{\mathrm{UV}} \sim-18 \pm 1$. The observed reduction in Ly $\alpha$ flux from galaxies at $z>6$ may be partly due to $f_{\mathrm{esc}}^{\text {ion }}$ approaching $f_{\text {esc,max }}^{\text {ion }}$ (also see Dijkstra et al. 2014). LAE clustering measurements and observations of Balmer lines can help determine the role of $f_{\mathrm{esc}}^{\text {ion }}$ in the disappearance of $\operatorname{Ly} \alpha$ emission from galaxies at $z \gtrsim 6$ (see Section 4.4).

4. Figure 2 also shows that the ionizing escape fraction is strongly affected by the cloud covering factor, $f_{\mathrm{cl}}$. As a result, $f_{\text {esc }}^{\text {ion }}$ is closely connected to the observed Ly $\alpha$ spectral line shape (see Section 4.3) with LyC-emitting objects typically having narrower, more symmetric Ly $\alpha$ lines (Figure 4, also see Erb et al. 2014). In multiphase models, LyC-emitting objects exhibit a wide range of spectral line profiles, and it is not possible to identify spectral features that "guarantee" a LyC detection.

Ly $\alpha$-emitting galaxies are valuable for constraining the ionization state of the intergalactic medium (see, e.g., Dijkstra et al. 2014, and references therein). Our work implies that these galaxies also provide unique insights into the nature of the sources that reionized the universe, in spite of the fact that modeling interstellar $\operatorname{Ly} \alpha$ radiative transfer remains highly challenging. We emphasize that our results differ from previous works that estimated the contribution of LAEs to cosmic reionization (see, e.g., Yajima et al. 2014): LAEs represent a subset of galaxies within a limited range of $M_{\mathrm{UV}}$ and with a (relatively) large $f_{\mathrm{esc}}^{\mathrm{Ly} \alpha}$ (and hence $f_{\mathrm{esc}}^{\mathrm{ion}}$ ), where the precise ranges in $M_{\mathrm{UV}}$ and $f_{\mathrm{esc}}^{\mathrm{Ly} \alpha}$ both depend on the minimum Ly $\alpha$ luminosity and $\mathrm{Ly} \alpha \mathrm{EW}$ of the LAE sample of interest. In addition, the contribution of LAEs to cosmic reionization depends sensitively on the pdf of the Ly $\alpha \mathrm{EW}$ as a function of $M_{\mathrm{UV}}$, which is not well constrained, especially at faint $M_{\mathrm{UV}}$. Here, we make a more general (and robust) point that the faint end of the LAE LF helps constrain the $M_{\mathrm{UV}}$ dependence of $f_{\text {esc }}^{\text {Ly } \alpha}$ and, by extension, $f_{\text {esc }}^{\text {ion }}$.

In the near future, the number of Ly $\alpha$-emitting galaxies at $z \sim 5.7-7$ is anticipated to grow by $\sim 1-2$ orders of magnitude with surveys performed on Subaru's Hyper Suprime-Cam (HSC). Moreover, integral-field unit spectrographs such as MUSE $^{15}$ will enable us to detect fainter Ly $\alpha$-emitting sources and better constrain the faint-end slope of the luminosity function of Ly $\alpha$ emitters, and also better characterize the (sometimes spatially resolved) spectra of $\operatorname{Ly} \alpha$ emission lines. Recent spectroscopic observations of gravitationally lensed galaxies (e.g., Treu et al. 2015; Schmidt et al. 2016; Vanzella et al. 2016a) have uncovered several (intrinsically) UV-faint galaxies with a prominent $\operatorname{Ly} \alpha$ emission line and/or other spectral features such as a high line ratio [O III] $\lambda 5007 /[\mathrm{O}$ II] $\lambda 3727$, which favor LyC escape (Huang et al. 2016; Vanzella et al. 2016a). These observations-which support the case for an enhanced contribution of UV-faint galaxies to cosmic reionization-provide a preview of what will be routinely possible with the next generation of ground-based telescopes such as the European Extremely Large Telescope (E-ELT) ${ }^{16}$, the Thirty Meter Telescope ${ }^{17}$, and the Giant Magellan Telescope. ${ }^{18}$

We thank the Aspen Center for Physics, where part of this collaboration was initiated, and which is supported by National Science Foundation grant PHY-1066293. M.D. thanks the

\footnotetext{
15 https://www.eso.org/sci/facilities/develop/instruments/muse.html

16 https://www.eso.org/sci/facilities/eelt/

17 http://www.tmt.org/

18 http://www.gmto.org/
} 
Table 1

Overview of the Model Parameters

\begin{tabular}{lccc}
\hline \hline Parameter & Fiducial Value & Allowed Range & Units \\
\hline$v_{\infty, \mathrm{cl}}$ & 100.0 & {$[0.0,800.0]$} & $\mathrm{km} \mathrm{s}^{-1}$ \\
$r_{\mathrm{cl}}$ & 100.0 & {$[30.0,200.0]$} & $\mathrm{pc}$ \\
$P_{\mathrm{cl}}$ & 0.35 & {$[0.0,1.0]$} & $\cdots$ \\
$H_{\mathrm{em}}$ & 1000.0 & {$\left[500.0,3.0 \times 10^{3}\right]$} & $\mathrm{pc}$ \\
$f_{\mathrm{cl}}$ & 3.5 & {$[0.8,8.0]$} & $\cdots$ \\
$T_{\mathrm{ICM}}{ }^{\dagger}$ & $10^{6}$ & {$\left[3.0 \times 10^{5}, 5.0 \times 10^{7}\right]$} & $\mathrm{K}$ \\
$n_{\mathrm{H} \mathrm{I}, \mathrm{ICM}}{ }^{\dagger}$ & $5.0 \times 10^{-8}$ & {$\left[10^{-12}, 10^{-6}\right]$} & $\mathrm{cm}^{-3}$ \\
$\sigma_{\mathrm{i}}$ & 50.0 & {$[5.0,100.0]$} & $\mathrm{km} \mathrm{s}^{-1}$ \\
$\zeta_{Z^{\dagger}}^{\dagger}$ & 0.01 & {$\left[10^{-4}, 0.1\right]$} & $\ldots$ \\
$T_{\mathrm{cl}}^{\dagger}$ & $10^{4}$ & {$\left[5.0 \times 10^{3}, 5.0 \times 10^{4}\right]$} & $\mathrm{K}$ \\
$\beta_{\mathrm{cl}}$ & 1.5 & {$[1.1,2.5]$} & $\cdots$ \\
$Z_{\mathrm{cl}}^{\dagger}$ & 0.2 & {$[0.03,1.0]$} & $Z_{\odot}$ \\
$\sigma_{\mathrm{cl}}$ & 40.0 & {$[5.0,100.0]$} & $\mathrm{km} \mathrm{s}^{-1}$ \\
$n_{\mathrm{H} \mathrm{I,cl}}^{\dagger}$ & 0.35 & {$[0.03,3.0]$} & $\mathrm{cm}^{-3}$ \\
\hline
\end{tabular}

Note. Variables marked with ${ }^{\dagger}$ were drawn from a uniform distribution in $\log$ space.

astronomy department at UCSB for their kind hospitality. M.G. thanks the Physics \& Astronomy department of JHU for their kind hospitality. A.V. gratefully acknowledges support from the University of San Francisco Faculty Development Fund. We thank Peng Oh and Crystal Martin for helpful discussions. Finally, we thank an anonymous referee for a constructive report that helped us improve the presentation of this work.

\section{APPENDIX \\ MODEL PARAMETERS}

Table 1 provides an overview of the 14 parameters that are needed to fully characterize the multiphase media. The second row contains the fiducial value for each parameter, which was taken from Laursen et al. (2013). The third row indicates the range of values from which we randomly drew model parameters.

\section{REFERENCES}

Adams, T. F. 1975, ApJ, 201, 350

Ahn, S.-H., Lee, H.-W., \& Lee, H. M. 2003, MNRAS, 340, 863

Alavi, A., Siana, B., Richard, J., et al. 2014, ApJ, 780, 143

Atek, H., Kunth, D., Hayes, M., Östlin, G., \& Mas-Hesse, J. M. 2008, A\&A, 488, 491

Barnes, L. A., \& Haehnelt, M. G. 2010, MNRAS, 403, 870

Becker, G. D., \& Bolton, J. S. 2013, MNRAS, 436, 1023

Behrens, C., Dijkstra, M., \& Niemeyer, J. C. 2014, A\&A, 563, A77

Benson, A., Venkatesan, A., \& Shull, J. M. 2013, ApJ, 770, 76

Bonilha, J. R. M., Ferch, R., Salpeter, E. E., Slater, G., \& Noerdlinger, P. D. 1979, ApJ, 233, 649

Borthakur, S., Heckman, T. M., Leitherer, C., \& Overzier, R. A. 2014, Sci, 346,216

Bouwens, R. J., Illingworth, G. D., Oesch, P. A., et al. 2012, ApJ, 754, 83

Bouwens, R. J., Illingworth, G. D., Oesch, P. A., et al. 2015, ApJ, 803, 34

Bouwens, R. J., Smit, R., Labbe, I., et al. 2015, arXiv:1511.08504

Bouwens, R. J., Smit, R., Labbe, I., et al. 2016, ApJ, in press (arXiv:1511. 08504)

Caruana, J., Bunker, A. J., Wilkins, S. M., et al. 2012, MNRAS, 427, 3055

Caruana, J., Bunker, A. J., Wilkins, S. M., et al. 2014, MNRAS, 443, 2831

Cen, R. 2003, ApJ, 591, 12

Chonis, T. S., Blanc, G. A., Hill, G. J., et al. 2013, ApJ, 775, 99

Conroy, C., \& Kratter, K. M. 2012, ApJ, 755, 123

Dijkstra, M. 2014, PASA, 31, e040

Dijkstra, M., \& Kramer, R. 2012, MNRAS, 424, 1672

Dijkstra, M., \& Loeb, A. 2008, MNRAS, 391, 457
Dijkstra, M., Wyithe, S., Haiman, Z., Mesinger, A., \& Pentericci, L. 2014, MNRAS, 440, 3309

Dove, J. B., Shull, J. M., \& Ferrara, A. 2000, ApJ, 531, 846

Dressler, A., Henry, A., Martin, C. L., et al. 2015, ApJ, 806, 19

Erb, D. K., Steidel, C. C., Trainor, R. F., et al. 2014, ApJ, 795, 33

Faisst, A. L. 2016, ApJ, submitted (arXiv:1605.06507)

Fernandez, E. R., \& Shull, J. M. 2011, ApJ, 731, 20

Ferrara, A., \& Loeb, A. 2013, MNRAS, 431, 2826

Finkelstein, S. L., Papovich, C., Ryan, R. E., et al. 2012a, ApJ, 758, 93

Finkelstein, S. L., Papovich, C., Salmon, B., et al. 2012b, ApJ, 756, 164

Fujita, A., Martin, C. L., Mac Low, M.-M., New, K. C. B., \& Weaver, R. 2009, ApJ, 698, 693

Gardner, J. P., Mather, J. C., Clampin, M., et al. 2006, SSRv, 123, 485

Gnedin, N. Y., Kravtsov, A. V., \& Chen, H.-W. 2008, ApJ, 672, 765

Gronke, M., Bull, P., \& Dijkstra, M. 2015a, ApJ, 812, 123

Gronke, M., \& Dijkstra, M. 2014, MNRAS, 444, 1095

Gronke, M., \& Dijkstra, M. 2016, ApJ, 826, 14

Gronke, M., Dijkstra, M., Trenti, M., \& Wyithe, S. 2015b, MNRAS, 449, 1284

Haiman, Z., \& Holder, G. P. 2003, ApJ, 595, 1

Hansen, M., \& Oh, S. P. 2006, MNRAS, 367, 979

Hashimoto, T., Verhamme, A., Ouchi, M., et al. 2015, ApJ, 812, 157

Hayes, M. 2015, PASA, 32, e027

Heckman, T. M., Borthakur, S., Overzier, R., et al. 2011, ApJ, 730, 5

Henry, A., Scarlata, C., Martin, C. L., \& Erb, D. 2015, ApJ, 809, 19

Huang, K., et al. 2016, ApJL, 823, L14

Inoue, A. K., Iwata, I., \& Deharveng, J.-M. 2006, MNRAS, 371, L1

Iwata, I., Inoue, A. K., Matsuda, Y., et al. 2009, ApJ, 692, 1287

Izotov, Y. I., Orlitová, I., Schaerer, D., et al. 2016, Natur, 529, 178

Jaacks, J., Thompson, R., \& Nagamine, K. 2013, ApJ, 766, 94

Jaskot, A. E., \& Oey, M. S. 2013, ApJ, 766, 91

Jensen, H., Hayes, M., Iliev, I. T., et al. 2014, MNRAS, 444, 2114

Jensen, H., Laursen, P., Mellema, G., et al. 2013, MNRAS, 428, 1366

Jones, T. A., Ellis, R. S., Schenker, M. A., \& Stark, D. P. 2013, ApJ, 779, 52

Kimm, T., \& Cen, R. 2014, ApJ, 788, 121

Konno, A., Ouchi, M., Nakajima, K., et al. 2016, ApJ, 823, 20

Kuhlen, M., \& Faucher-Giguère, C.-A. 2012, MNRAS, 423, 862

Kulas, K. R., Shapley, A. E., Kollmeier, J. A., et al. 2012, ApJ, 745, 33

Kunth, D., Mas-Hesse, J. M., Terlevich, E., et al. 1998, A\&A, 334, 11

Laursen, P., Duval, F., \& Ostlin, G. 2013, ApJ, 766, 124

Laursen, P., Sommer-Larsen, J., \& Andersen, A. C. 2009, ApJ, 704, 1640

Laursen, P., Sommer-Larsen, J., \& Razoumov, A. O. 2011, ApJ, 728, 52

Liu, C., Mutch, S. J., Angel, P. W., et al. 2016, MNRAS, 462, 235

Livermore, R. C., Finkelstein, S. L., \& Lotz, J. M. 2016, ApJ, submitted (arXiv:1604.06799)

Ma, X., Hopkins, P. F., Kasen, D., et al. 2016, MNRAS, 459, 3614

Ma, X., Kasen, D., Hopkins, P. F., et al. 2015, MNRAS, 453, 960

Mason, C. A., Trenti, M., \& Treu, T. 2015, ApJ, 813, 21

Mas-Ribas, L., \& Dijkstra, M. 2016, ApJ, 822, 84

Matthee, J., Sobral, D., Best, P., et al. 2016a, arXiv:1605.08782

Matthee, J., Sobral, D., Oteo, I., et al. 2016b, MNRAS, 458, 449

Matthee, J., Sobral, D., Santos, S., et al. 2015, MNRAS, 451, 400

McKee, C. F., \& Ostriker, J. P. 1977, ApJ, 218, 148

McQuinn, M., Hernquist, L., Zaldarriaga, M., \& Dutta, S. 2007, MNRAS, 381,75

Mesinger, A., \& Furlanetto, S. R. 2008, MNRAS, 386, 1990

Micheva, G., Iwata, I., \& Inoue, A. K. 2016, MNRAS, in press (arXiv:1604 00102)

Mitra, S., Choudhury, T. R., \& Ferrara, A. 2011, MNRAS, 413, 1569

Nakajima, K., \& Ouchi, M. 2014, MNRAS, 442, 900

Neufeld, D. A. 1991, ApJL, 370, L85

O'Shea, B. W., Wise, J. H., Xu, H., \& Norman, M. L. 2015, ApJL, 807, L12

Ono, Y., Ouchi, M., Mobasher, B., et al. 2012, ApJ, 744, 83

Oyarzún, G. A., Blanc, G. A., González, V., et al. 2016, ApJL, 821, L14

Paardekooper, J.-P., Khochfar, S., \& Dalla Vecchia, C. 2013, MNRAS, 429, L94

Paardekooper, J.-P., Pelupessy, F. I., Altay, G., \& Kruip, C. J. H. 2011, A\&A, 530, A87

Parsa, S., Dunlop, J. S., McLure, R. J., \& Mortlock, A. 2016, MNRAS, 456, 3194

Pei, Y. C. 1992, ApJ, 395, 130

Pentericci, L., Fontana, A., Vanzella, E., et al. 2011, ApJ, 743, 132

Pentericci, L., Vanzella, E., Fontana, A., et al. 2014, ApJ, 793, 113

Rauch, M., Becker, G. D., Haehnelt, M. G., et al. 2011, MNRAS, 418, 1115

Rauch, M., Haehnelt, M., Bunker, A., et al. 2008, ApJ, 681, 856

Reddy, N. A., \& Steidel, C. C. 2009, ApJ, 692, 778

Rivera-Thorsen, T. E., Hayes, M., Östlin, G., et al. 2015, ApJ, 805, 14 
Robertson, B. E., Furlanetto, S. R., Schneider, E., et al. 2013, ApJ, 768, 71 Schaerer, D. 2003, A\&A, 397, 527

Schenker, M. A., Stark, D. P., Ellis, R. S., et al. 2012, ApJ, 744, 179

Schmidt, K. B., Treu, T., Bradač, M., et al. 2016, ApJ, 818, 38

Sharma, M., Theuns, T., Frenk, C., et al. 2016, MNRAS, 458, L94

Shibuya, T., Ouchi, M., Nakajima, K., et al. 2014, ApJ, 788, 74

Shull, J. M., Harness, A., Trenti, M., \& Smith, B. D. 2012, ApJ, 747, 100

Smith, B. M., Windhorst, R. A., Jansen, R. A., et al. 2016, ApJ, submitted (arXiv:1602.01555)

Sobacchi, E., \& Mesinger, A. 2015, MNRAS, 453, 1843

Song, M., Finkelstein, S. L., Gebhardt, K., et al. 2014, ApJ, 791, 3

Stark, D. P., Ellis, R. S., Chiu, K., Ouchi, M., \& Bunker, A. 2010, MNRAS, 408, 1628

Steidel, C. C., Erb, D. K., Shapley, A. E., et al. 2010, ApJ, 717, 289

Trainor, R. F., Steidel, C. C., Strom, A. L., \& Rudie, G. C. 2015, ApJ, 809, 89

Treu, T., Schmidt, K. B., Brammer, G. B., et al. 2015, ApJ, 812, 114

Vanzella, E., De Barros, S., Cupani, G., et al. 2016a, ApJL, 821, L27
Vanzella, E., de Barros, S., Vasei, K., et al. 2016b, ApJ, 825, 41

Verhamme, A., Orlitová, I., Schaerer, D., \& Hayes, M. 2015, A\&A, 578, A7

Verhamme, A., Schaerer, D., Atek, H., \& Tapken, C. 2008, A\&A, 491, 89

Verhamme, A., Schaerer, D., \& Maselli, A. 2006, A\&A, 460, 397

Verner, D. A., Ferland, G. J., Korista, K. T., \& Yakovlev, D. G. 1996, ApJ, 465,487

Wilkins, S. M., Bunker, A. J., Lorenzoni, S., \& Caruana, J. 2011, MNRAS, 411, 23

Wise, J. H., Demchenko, V. G., Halicek, M. T., et al. 2014, MNRAS, 442,2560

Wofford, A., Leitherer, C., \& Salzer, J. 2013, ApJ, 765, 118

Wyithe, J. S. B., \& Loeb, A. 2003, ApJ, 586, 693

Yajima, H., Choi, J.-H., \& Nagamine, K. 2011, MNRAS, 412, 411

Yajima, H., \& Li, Y. 2014, MNRAS, 437, 3962

Yajima, H., Li, Y., Zhu, Q., et al. 2014, MNRAS, 440, 776

Yang, H., Malhotra, S., Gronke, M., et al. 2016, ApJ, 820, 130 